\title{
Riesgos ambientales y su impacto en la mortalidad por insuficiencia renal crónica en el estado de Tlaxcala México
}

Environmental risk and therir impact on mortality by chronic renal failure in Tlaxcala State, Mexico

\author{
García-Juárez Guillermina ${ }^{1 凶}$, Hernández-Vázquez Maricela ${ }^{1}$, Orozco-Bolaños Hermila ${ }^{1}$, Suárez-González \\ Gerardo $^{3}$, Ramírez-Bribiesca Efrén ${ }^{4}$, Juárez-Ortiz María Guadalupe ${ }^{1}$. \\ ${ }^{1}$ Universidad Autónoma de Tlaxcala. Facultad de Agrobiología. Km 10.5 Autopista Tlaxcala-San Martín \\ Texmelucan, Ixtacuixtla, Tlax. C.P. 90120. \\ Colegio de Tlaxcala A. C., México. \\ Colegio de Posgraduados, Campus Montecillo. Texcoco, Estado de México. \\ ${ }^{凶}$ Autor para correspondencia: nefertiti_ggj58@yahoo.com
}

Recibido: $20 / 03 / 2018$

Aceptado: 24/05/2018

\section{RESUMEN}

Se analizaron los factores de riesgo ambiental y su impacto en la mortalidad por insuficiencia renal crónica (IRC) en el estado de Tlaxcala. El estudio fue transversal-analítico, se obtuvieron registros de mortalidad por (IRC) del periodo 2012-2013, sitios potencialmente contaminantes y metales pesados en tres fuentes de información. Se observó que el municipio más afectado por mortalidad fue el Carmen Tequexquitla con una tasa de mortalidad de 45.54 por 100,000 habitantes. El municipio con más sitios potencialmente contaminantes fue Huamantla, ubicado en la región oriente. Hubo correlación entre Arsénico y mortalidad por (IRC) con una diferencia significativa de $\mathrm{p} \leq 0.05$. Entre los riesgos ambientales y mortalidad hubo significancia de $\mathrm{p} \leq 0.05$. La asociación entre estas variables corroboró que la presencia de factores de riesgo ambiental, influyen en la mortalidad por (IRC), principalmente en los municipios localizados en la región oriente del estado.

Palabras clave: riesgos, ambientales, mortalidad, insuficiencia renal crónica

\begin{abstract}
It was analized the environmental risk factors and their impact on the mortality by chronic renal failure (CRF) in Tlaxcala State. The study was analytical-transverse, it was obtained mortality data by (CRF) from 2012 to 2013, from potentially polluted places and heavy metal in three sources of information. It was observed that the town more affected by mortality was the El Carmen Tequexquitla with a mortality rate of 45.54 per each 10,0000 habitants. The town with more contaminant places is Huamantla, located in the orient region. There is a correlation between Arsenic and mortality by (CRF) with a significative difference of $p \leq 0.05$. Between ambiental risks and mortality there are a significance
\end{abstract}


of $\mathrm{p} \leq 0.05$. The association between the variables confirm that the ambiental risk factors presence has influence on the mortality by (CFR), mainly in the towns located in the orient region of the state.

Key words: risks, environmental, mortality, chronic renal failure

\section{INTRODUCCIÓN}

La protección ambiental y reducción de los efectos nocivos al ambiente y salud son un objetivo en el proceso de desarrollo económico y social (Ordóñez, 2000). El campo de la salud ambiental, se vincula con la salud humana y calidad de vida, por la influencia de factores físicos, químicos, biológicos, y psicosociales en esta. En general las industrias desechan diferentes sustancias químicas al medio ambiente, alterando la calidad de la atmósfera, suelo, agua, flora, fauna e interacciones entre estos elementos (Fernández, 2012). Desde 1998, la Organización Mundial de la Salud, presentó las prioridades sobre la prevención y protección ambiental de las diferentes sustancias tóxicas, por constituir factores de riesgo potencial al ambiente y salud, intensificados a partir de la revolución industrial, amenazando los ecosistemas y desarrollo sustentable (Méndez, 2010 y Leff, 2012), por ello es importante difundir la necesidad de la conservación del entorno, que asegure el equilibrio ecológico, y la salud pública (Diario Oficial de la Federación, 2004).

La presencia de residuos tóxicos como los metales pesados, impactan en la salud ambiental y humana, provienen principalmente de diferentes fuentes como la industria, tiraderos de residuos, zonas agrícolas entre otras, se depositan tanto en suelos, agua, aire, alimentos animales y vegetales (López, 2005 y Rengifo, 2008). La exposición ambiental a metales pesados como el Cadmio (Cd), Plomo $(\mathrm{Pb})$ y Arsénico (As) ocurre por diferentes mecanismos y se asocia con daños orgánicos como la insuficiencia renal en el hombre (Calzada, 2007 y Sabath, 2012).

La Insuficiencia Renal Crónica (IRC), comprende la pérdida progresiva e irreversible de las funciones renales por más de tres meses, en general la exposición crónica a $\mathrm{Cd}, \mathrm{Pb}$ y As, se asocia con nefropatía a nivel de estructuras anatómicas a nivel glomerular y túbulo contorneado proximal con daño progresivo de la función renal (Klassen, 2004 y Huang et. al.,2009). La IRC, cuando se manifiesta clínicamente es porque ya existe un grado de afectación irreversible en las células glomerulares y función de las estructuras renales (Thévenod, 2010).

El registro de mortalidad por IRC en el estado de Tlaxcala, ha ocupado los primeros sitios a nivel nacional (INEGI, SESA, CONAPO, 2015), afectando principalmente a los niños y población joven entre los 15-24 años (Fernández, 2006). En la población derechohabiente del Instituto Mexicano del Seguro Social (IMSS), Hospital General de Zona 1 Tlaxcala, se observó que la población en diálisis por IRC, el grupo más afectado es entre 10-19 años (Ruíz, 2010), representando alto costo económico para su tratamiento por diálisis, hemodiálisis y trasplantes, con una evolución incierta, daño irreversible y muerte inminente, con pérdida de población potencialmente productiva (Inserra, et al., 2007 y Alva, 2010). La Secretaría de salud (2014), reportó 272 casos de mortalidad por IRC en el estado de Tlaxcala, durante el periodo 2012- 
2013. No obstante que el panorama epidemiológico de la IRC es multicausal, está influenciado por la exposición a metales pesados (Sabath, 2012) y otros riesgos ambientales por lo que el supuesto de esta investigación es que los municipios que presentan mayor mortalidad por IRC, están asociados a la presencia de factores de riesgo ambiental. En Tlaxcala, no existen estudios relacionados con riesgos ambientales y daño renal, por lo que el objetivo es correlacionar y asociar estas variables.

\section{MATERIAL Y MÉTODOS}

El estudio fue transversal-analítico, las variables fueron: 1.Factores de riesgo ambiental, cuyo análisis fue a) identificar y regionalizar los sitios potencialmente contaminantes con información en el estado de Tlaxcala (López, 2005), tomando en cuenta la presencia de industrias, microindustrias, industrias agrícolas, rellenos sanitarios, rastros y gaseras; b) se obtuvo información, analizó y regionalizó la presencia de metales pesados en suelos (Calzada, 2007), el trabajo se enfocó al ( $\mathrm{Cd}, \mathrm{Pb}$ y $\mathrm{As}$ ), por su importancia de provocar daño renal ; 2. Mortalidad por IRC, esta información fue proporcionada por la Subdirección de Promoción a la Salud a nivel federal, del periodo 2012-2013 los registros se analizaron y calcularon las tasas de mortalidad por cada 100.000 habitantes, en el estado de Tlaxcala, INEGI (2010). Se identificaron los municipios con mayor mortalidad (Figura1), excluyéndose los municipios de Tzompantepec, Tlaxcala capital, y Calpulalpan, por contar con un hospital regional con unidades de atención a insuficientes renales, condición que concentra pacientes en estadios finales, provenientes de diferentes zonas, y ahí mismo mueren, siendo un sesgo para la mortalidad real en estos lugares. El análisis estadístico, fue de correlación con $\mathrm{r}$ de Pearson y de asociación con apoyo del programa SPSS, versión 20, entre la mortalidad por IRC y riesgos ambientales. Para la regionalización de variables se utilizó el programa ArcGis 1.5

Figura 1. Mortalidad por insuficiencia renal en el estado de Tlaxcala 2012-2013.

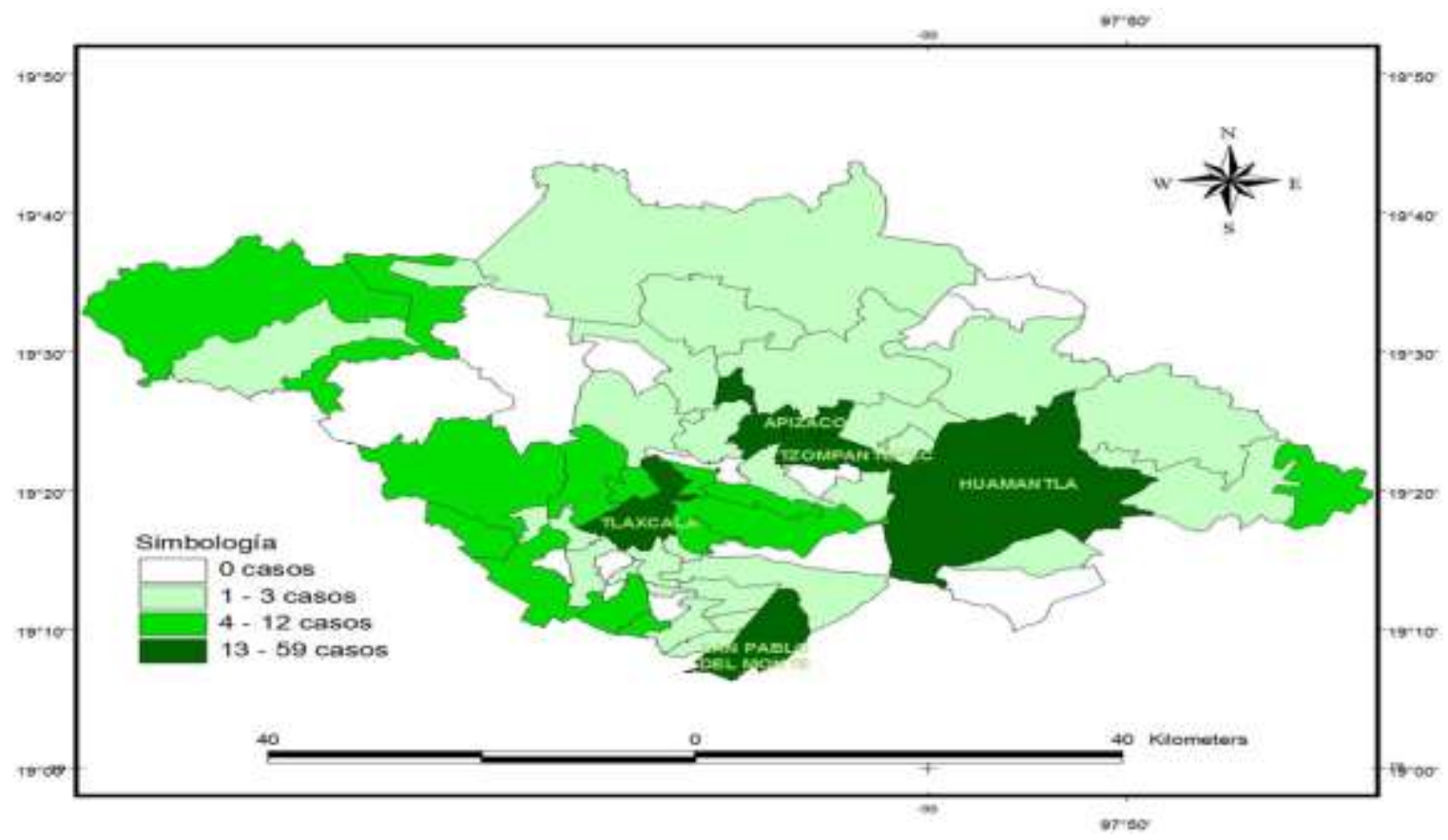




\section{RESULTADOS}

\section{Factores de riesgo}

\section{a). Sitios potencialmente contaminantes}

Al analizar la información en el estado de Tlaxcala, se encontró que hay un listado de 34 sitios, distribuidos en 22 municipios de 60, considerados potencialmente contaminantes, es decir el $36.6 \%$ de la entidad tiene algún tipo de riesgo ambiental. Respecto a la presencia de gaseras, rastros y rellenos sanitarios, los municipios con riesgo son Tetla de la Solidaridad, Apizaco y Yauhquemecan; Las industrias agrícolas se localizan en los municipios de Huamantla, Apizaco, y Tlaxcala capital; Las microindustrias se ubican en el municipio de Españita y San Pablo del Monte; Las zonas industriales (corredores) se encuentran distribuidas en diferentes municipios, observando que las localidades de El Carmen Tequexquitla, Huamantla y Apizaco presentan riesgos.

b). Presencia de metales pesados: Cadmio (Cd), Plomo (Pb) y Arsénico (As) en el estado de Tlaxcala

Los municipios donde se encontró $\mathrm{Cd}$ por arriba de los límites permisibles, fueron Atlangatepec, Altzayanca, Huamantla, Ixtacuixtla, Teolocholco y San Francisco Tetlanohcan con dos décimas de $\mathrm{mg} / \mathrm{kg}$; el $\mathrm{Pb}$ se encontró en concentraciones normales; el As rebasó $27.58 \mathrm{mg} / \mathrm{kg}$ por arriba de los parámetros permitidos. Al regionalizar la presencia de metales pesados (Figura, 2) se observó que los municipios que rebasan el límite permisible de As son principalmente Huamantla, Altzayanca, El Carmen Tequexquitla, Cuapiaxtla e Ixtenco.

Figura 2. Presencia de Arsénico, Cadmio y Plomo en el estado de Tlaxcala

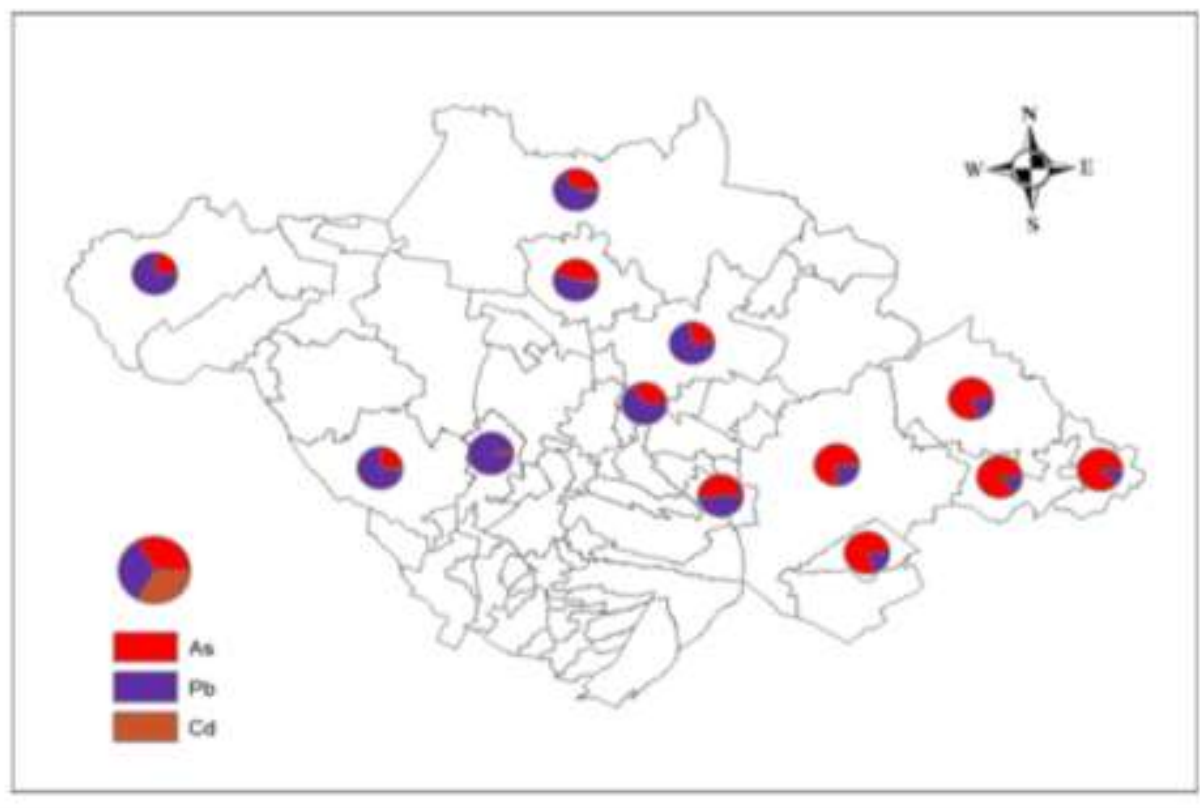

Para valorar la asociación entre metales pesados y la mortalidad, se realizó una prueba de chicuadrada para descartar la hipótesis nula de asociación entre ambas variables, teniendo un grado de significancia de .232 (Cuadro, 1), confirmando que de los metales pesados analizados el As influye en la mortalidad por IRC, en los municipios donde se reportó mayor concentración. 
Cuadro 1. Asociación entre Arsénico y mortalidad por insuficiencia renal crónica

\begin{tabular}{l|c|c|c}
\hline & Valor & Gl & Sig. asintótica (bilateral) \\
\hline Chi-cuadrado de Pearson & $110.000^{\mathrm{a}}$ & 100 & 0.232 \\
\hline Razón de verosimilitudes & 52.754 & 100 & 1.000 \\
\hline Asociación lineal por lineal & 3.665 & 1 & .056 \\
\hline $\mathrm{N}$ de casos válidos & 11 & & \\
\hline a. 121 casillas (100.0\%) tienen una frecuencia esperada inferior a 5. La frecuencia mínima \\
esperada es .09
\end{tabular}

\section{Mortalidad por insuficiencia renal crónica y riesgos ambientales}

El Cuadro 2, muestra los municipios con mayor mortalidad por IRC, El Carmen Tequexquitla,
Ixtenco, Huamantla, Apizaco, Panotla y Altzayanca; así como su relación con riesgos ambientales (sitios potencialmente contaminantes y niveles de metales pesados en suelo).

Cuadro 2. Municipios con mayor mortalidad por IRC y riesgos ambientales

\begin{tabular}{c|c|c|c|c|c|c}
\hline Municipios & $\begin{array}{c}\text { Tasa de } \\
\text { mortalidad/100,000 } \\
\text { habitantes }\end{array}$ & $\begin{array}{c}\text { Sitios } \\
\text { potencialmente } \\
\text { Contaminantes }\end{array}$ & $\begin{array}{c}\text { Arsénico } \\
(\mathrm{As}) \\
\mathrm{mg} / \mathrm{kg}\end{array}$ & $\begin{array}{c}\text { Cadmio } \\
(\mathrm{Cd}) \\
\mathrm{mg} / \mathrm{kg}\end{array}$ & $\begin{array}{c}\text { Plomo } \\
(\mathrm{Pb}) \mathrm{mg} / \mathrm{kg}\end{array}$ & $\begin{array}{c}\text { Total de } \\
\text { Riesgos } \\
(0-15)\end{array}$ \\
\hline Cuapiaxtla & 14.62 & 9 & 71.6 & 0.22 & 10.76 & 9 \\
\hline Atlangatepec & 16.61 & 11 & 8.03 & 0.7 & 9.38 & 11 \\
\hline Ixtacuixtla & 17.06 & 14 & 7.85 & 0.46 & 23.84 & 14 \\
\hline $\begin{array}{c}\text { San Josè } \\
\text { Teacalco }\end{array}$ & 17.66 & 3 & 13.57 & & & 3 \\
\hline Altzayanca & 18.82 & 10 & 66.83 & 0.47 & 15.87 & 10 \\
\hline Panotla & 19.89 & 12 & 0.38 & 0.13 & 9.36 & 12 \\
\hline Apizaco & 22.20 & 7 & 5.64 & 0.12 & 11.82 & 7 \\
\hline Huamantla & 25.88 & 15 & 41.83 & 0.57 & 13.20 & 15 \\
\hline Ixtenco & 44.17 & 11 & 77.82 & 0.87 & 18.05 & 11 \\
\hline $\begin{array}{c}\text { El Carmen } \\
\text { Tequexquitla }\end{array}$ & 45.54 & 10 & 85.39 & 0.47 & 12.04 & 10 \\
\hline
\end{tabular}

El Cuadro 3, presenta la correlación entre las variables: metales pesados, riesgos ambientales y mortalidad por IRC, se evidencia una correlación estadísticamente significativa de $\mathrm{p} \leq$
0.05 entre la tasa de mortalidad, concentración de As, así como por la presencia de riesgos ambientales. 
Cuadro 3. Coeficientes de correlación $\mathrm{As}, \mathrm{Cd}, \mathrm{Pb}$, riesgos ambientales (RA) y tasas de mortalidad

\begin{tabular}{|c|c|c|c|c|c|c|}
\hline & & $\begin{array}{c}\text { Tasa de } \\
\text { mortalidad }\end{array}$ & As & Cd & $\mathbf{P b}$ & RA \\
\hline \multirow{6}{*}{ Tasa de mortalidad } & Correlación de Pearson & 1 & $.605^{*}$ & .476 & .115 & .118 \\
\hline & Sig. (bilateral) & & .048 & .139 & .737 & .731 \\
\hline & Suma de cuadrados y & 1154.984 & 2223.199 & 14.847 & 51.641 & 41.636 \\
\hline & productos cruzados & & & & & \\
\hline & Covarianza & 115.498 & 222.320 & 1.485 & 5.164 & 4.164 \\
\hline & $\mathrm{N}$ & 11 & 11 & 11 & 11 & 11 \\
\hline \multirow{6}{*}{ As } & Correlación de Pearson & $.605^{*}$ & 1 & .493 & .054 & .012 \\
\hline & Sig. (bilateral) & .048 & & .123 & .875 & .972 \\
\hline & Suma de cuadrados y & 2223.199 & 11674.890 & 48.950 & 77.173 & 13.528 \\
\hline & productos cruzados & & & & & \\
\hline & & 222.320 & 1167.489 & 4.895 & 7.717 & 1.353 \\
\hline & $\mathrm{N}$ & 11 & 11 & 11 & 11 & 11 \\
\hline \multirow{6}{*}{$\mathrm{Cd}$} & Correlación de Pearson & .476 & .493 & 1 & .279 & .469 \\
\hline & Sig. (bilateral) & .139 & .123 & & .405 & .146 \\
\hline & Suma de cuadrados y & 14.847 & 48.950 & .843 & 3.405 & 4.482 \\
\hline & productos cruzados & & & & & \\
\hline & Covarianza & 1.485 & 4.895 & .084 & .340 & .448 \\
\hline & $\mathrm{N}$ & 11 & 11 & 11 & 11 & 11 \\
\hline \multirow{6}{*}{$\mathrm{Pb}$} & Correlación de Pearson & .115 & .054 & .279 & 1 & .338 \\
\hline & Sig. (bilateral) & .737 & .875 & .405 & & .309 \\
\hline & Suma de cuadrados y & 51.641 & 77.173 & 3.405 & 176.006 & 46.709 \\
\hline & productos cruzados & & & & & \\
\hline & Covarianza & 5.164 & 7.717 & .340 & 17.601 & 4.671 \\
\hline & $\mathrm{N}$ & 11 & 11 & 11 & 11 & 11 \\
\hline \multirow{6}{*}{ RA } & Correlación de Pearson & .118 & .012 & .469 & .338 & 1 \\
\hline & Sig. (bilateral) & .731 & .972 & .146 & .309 & \\
\hline & Suma de cuadrados y & 41.636 & 13.528 & 4.482 & 46.709 & 108.545 \\
\hline & productos cruzados & & & & & \\
\hline & Covarianza & 4.164 & 1.353 & .448 & 4.671 & 10.855 \\
\hline & $\mathrm{N}$ & 11 & 11 & 11 & 11 & 11 \\
\hline
\end{tabular}

Se corroboró que los riesgos ambientales inciden en la mortalidad por insuficiencia renal (Cuadro, IV), en los municipios de Huamantla,
Ixtacuixtla, Panotla, Atlangatepec, Ixtenco y el Carmen Tequexquitla. 
Cuadro IV. Asociación entre riesgos ambientales y tasa de mortalidad por insuficiencia renal crónica (IRC)

\begin{tabular}{l|l|l|l}
\hline & Valor & Gl & $\begin{array}{l}\text { Sig. asintótica } \\
\text { (bilateral) }\end{array}$ \\
\hline Chi-cuadrado de Pearson & $77.000^{\mathrm{a}}$ & 70 & .265 \\
\hline Razón de verosimilitudes & 44.436 & 70 & .993 \\
\hline Asociación lineal por lineal & .138 & 1 & .710 \\
\hline $\mathrm{N}$ de casos válidos & 11 & & \\
\hline
\end{tabular}

88 casillas (100.0\%) tienen una frecuencia esperada inferior a 5. La frecuencia mínima esperada es .09

\section{DISCUSIÓN}

\section{Factores de riesgo ambiental}

\section{a). Sitios potenciales de contaminación ambiental}

De acuerdo a lo encontrado en este trabajo sobre el efecto que tienen los factores de riesgo ambiental, se encontró una asociación con la mortalidad por IRC coincidiendo con otra investigación donde señala, que históricamente a nivel mundial, desde la Revolución Industrial se han incrementado diferentes sustancias químicas amenazando al ambiente y salud del hombre (Lin et. al., 2001).

Respecto a los factores de riesgo ambiental (Ortiz et al., 2014), señalan como peligros potenciales a las industrias, las cuales emiten metales pesados en suelos y agua principalmente, afectando cadenas tróficas, amenazando la salud pública con el desarrollo de enfermedades crónico degenerativas, como la IRC, hallazgo relacionados con lo encontrado en este estudio.

\section{Metales pesados}

Para (Treviño, 2004 y Galán et. al., 2008), la contaminación ambiental es un riesgo que impacta en la salud del hombre, principalmente por metales pesados como $\mathrm{Cd}, \mathrm{Pb}$ y $\mathrm{As}$ con importantes niveles de toxicidad lo que amenazan la salud del hombre, en particular a órganos como el riñón.

La presencia de metales pesados, son de interés normativo (Diario Oficial de la Federación, 2004), este documento señala que el objetivo es prevenir los efectos tóxicos de estos elementos a la flora, fauna y al hombre, considera que los límites permisibles en suelos de uso agrícola, residencial o comercial deben ser para el As de 22; Cd 37 y $\mathrm{Pb} 400 \mathrm{mg} / \mathrm{kg}$ respectivamente, estos parámetros permitieron observar en esta investigación que los niveles del As, en algunos municipios resultaron a concentraciones hasta $27.58 \mathrm{mg} / \mathrm{kg}$ por arriba de los niveles aceptables y se asoció a la muerte por IRC.

\section{Mortalidad por Insuficiencia Renal Crónica (IRC)}

Sobre el tiempo de exposición con metales pesados provenientes de procesos industriales, suelos contaminados, mantos acuíferos, alimentos agrícolas y agua destinada para la 
ingesta humana, se consideran factores predisponentes para el daño renal, su evolución y pronóstico. En Estados Unidos de Norteamérica, U.S Renal Data System (USRDS, 2009), observó un incremento de pacientes renales crónicos del $125 \%$ de pacientes de 1991 a 2004, que no mejoran con diálisis, por lo que requieren de un trasplante para poder sobrevivir, esta condición es importante ya que el estado de Tlaxcala presenta situaciones epidemiológicas similares emergentes, que amenazan con el incremento de mortalidad por IRC, asociada a diferentes riesgos entre ellos los ambientales.

El resultado de esta investigación en el estado de Tlaxcala, sobre la asociación entre el As y la mortalidad por IRC, concuerda con otros estudios (Lytle, 2004 y Mahmudur, 2009, Reyes et al., 2016) es uno de los contaminantes inorgánicos más abundantes y millones de personas están expuestas por diferentes mecanismos, propiciando el desarrollo de la IRC.

\section{CONCLUSIÓN}

Los municipios que cuentan con mayores factores de riesgo ambiental por contar con sitios potencialmente contaminante son: El Carmen Tequexquitla, Huamantla e Ixtenco, localizados en la región Oriente del estado de Tlaxcala.

El As y el Cd rebasan los niveles normales, sin embargo solo se encontró correlación y asociación entre el As y la IRC en los municipios de El Carmen Tequexquitla, Ixtenco, Huamantla, Altzayanca y Cuapiaxtla, localizados en la región Oriente.

Los hallazgos de esta investigación muestran un problema importante de contaminación en suelos por metales pesados y sitios potencialmente peligrosos, lo que sugiere líneas de investigación para determinar la presencia de estos en agua de consumo humano, alimentos agrícolas como amenazas para la salud pública.

\section{LITERATURA CITADA}

Alva G.B. 2010. Condicionantes del daño renal enfermedades glomerulares. En Universidad Autónoma de Tlaxcala. XVI Jornadas Médicas Interinstitucionales, 5/6 noviembre, 2010. Tlaxcala, México

Calzada J.M. Mapas geoquímicos de metales pesados de metales pesados de suelos del Estado de Tlaxcala, México [tesis de maestría]. Ciencias de la Tierra, Universidad Nacional Autónoma de México. 2007.

Diario Oficial de la Federación NOM-147SEMARNAT/SSA1-2004. Criterios para determinar las concentraciones de remediación de suelos contaminados por arsénico, bario, berilio, cadmio, cromo hexavalente, mercurio, níquel, plata, plomo, selenio, talio y/o vanadio. México: Diario Oficial de la Federación, 2007[consultado el 9 de julio de 2016].

Disponible en: http://www.profepa.gob.mx/innovaportal/ file/1392/1/nom-147-semarnat_ssa12004.pdf

Fernández C.S.2006. El IMSS en cifras: la demanda de servicios en urgencias. Rev Med Inst Mex Seg Soc. 2006; 44 (3):261273.

http://www.redalyc.org/articulo.oa?id=45 7745540004

Fernández SP. El Informe Brundtland, 19872012. Responsabilidad Social para el Desarrollo Sostenible, México; 2012. https://doi.org/10.1016/j.gexplo.2008.01.0 Galán. E., Fernández. C.J.C., González I., Aparicio P., Romero A. 2008. Influence of geological setting of geochemical 
baselines or trace elements in solis. Application to solis of south-west Spain, en Journal of Geochemical Exploration 98; 89-106 www.elsevier.com/locate/jgeoexp

Huang M., Choiu S.J., Kim N.Y., Park C.H. 2009. Risk assessment of low-level cadmiun and arsenic on the kidney. In Journal Toxicol Environ Health. 72:14938. https://doi.org/10.1080/152873909032

INEGI, SESA, CONAPO. Causas de muerte en México 2006. (sitio en internet]. 2006. [consultado el 23 de enero de 2015]. Disponible en: http: //sexto.informe.fox.presidencia.gob.mx/d ocs/anexo/pdf/P102.pdf

INEGI. Censo de Población y Vivienda.2010. Tlaxcala/ Población/ Población total por municipio [sitio en internet]. [consultado el 20 de diciembre de 2016]. Disponible en:

www.beta.inegi.org.mx/proyectos/ccpv/2 010/

Inserra F, De La Llave G., Alpino M., Castagna R., De La Fuente D.E. 2007. Relevamiento de factores de riesgo y de enfermedad renal en familiares de pacientes en diálisis. Medicina [Internet]. [citado 18 de enero de 2017]; 67(1): 8-18 Disponible

en: www.scielo.org.ar/pdf/medba/v67n1/v67 n1a02.pdf

Klassen R.B. 2004. Megalin mediates renal uptake of heavy metal methallothionein complex. An Am J. Physiol Renal Physiol. 287(3): 393-403. https://doi.org/10.1152/ajprenal.00233.200

Leff E.2012. Discursos sustentables. México: Siglo XXI, editores.

Lin J.L., Yu C.C., Lin-Tan D., Ho H.H. 2001. Lead chelation therapy and urate excretion in patients with choronic renal disease and gout. an Kidney International [Internet] 2001 [citado el 18 de agosto de 2016] Vol. 60 : 266-71.

https://doi.org/10.1046/j.1523-1755.2001.

López S.J.G., Sánchez A.J, Valencia Q.R., Juárez L., Pérez G.L., Ortiz O.E., Zempoalteca E.S. 2005. Elaboración de un padrón e inspección de sitios potencialmente peligrosos en el estado de Tlaxcala y sus impactos en la salud. En: $\mathrm{X}$ Congreso Nacional y IV Internacional de Ciencias Básicas. Rev. Int. Contam. Ambient, Volumen 21 (jun 2005). Quintana Roo, México.

Lytle D, Sorg T.H. 2004. Accumulation of Arsenic in Drinking Water Distribution Systems. U.S. Environmental Protection. In Environ. Sci. Technol. 38(20): 53655372.

https://doi.org/10.1021/es049850v

Mahmudur R. 2009. Chronic exposure of arsenic via drinking water and its adverse health impacts on humans. In Environ Geochem Health. Apr;31 Suppl 1:189200. https://doi.org/10.1007/s10653-008

Méndez D.A., Méndez B.F.., Tapia YT., Muñoz M.A, Aguilar S.L.2010. Epidemiología de la insuficiencia renal crónica en México. En Diálisis y Trasplante, 31(1):7-11. https://doi.org/10.1016/S1886-2845(10)70

Nava R.C., Méndez A.M. 2011. Efectos neurotóxicos de metales pesados (cadmio, plomo, arsénico y talio). Archivos de Neurociencias, 16(3):140-147. http://www.medigraphic.com/pdfs/arcneu /ane-2011/ane113f.pdf

Ordóñez G.A. 2007. Salud Ambiental, conceptos y actividades. Rev Panam 
Salud Publica/Pan Am J Public Health, 7(3): 137-147. https://doi.org/10.1590/S1020-498920000

Ortiz H.M.L., Sánchez S.E., Folch M.J.L, Olvera V.A., Dantán G.E. 2014. Plaguicidas: Generalidades usos e impactos sobre el ambiente y la salud. $1^{\text {a }}$ edic. Cuernavaca, Morelos. Universidad Autónoma del Estado de Morelos, México.

Rengifo C.H. 2008. Conceptualización de la salud ambiental: teoría y práctica. en Rev. Perú. Med. Exp Salud pública [Internet]. [citado 4 de abril de 2016]; 25(4): 403-9. Disponible en http://www.scielo.org.pe/scielo.php?scrip $\mathrm{t}=\mathrm{sci}$ _arttext\&pid=S172646342008000400010

Reyes Y., Vergara I., Torres E.O., Díaz M., González, E. 2016. Contaminación por metales pesados: implicaciones en salud, ambiente y seguridad alimentaria. en Revista Ingeniería, Investigación y Desarrollo. 16(2): 66-77 https://doi.org/10.19053/1900771X.v16.n2
Ruíz T.M.L 2010. Panorama epidemiológico del daño renal. En XVI Jornadas Médicas Interinstitucionales. Universidad Autónoma de Tlaxcala, 5 y 6 de noviembre de 2010. Tlaxcala, México.

Sabath E. 2012. Medio ambiente y riñón: nefrotoxicidad por metales pesados. en Rev. Nefrología. 32: 279-86. doi:10.3265/Nefrologia.pre2012.Jan.1092 8

Secretaría de Salud. 2014. Mortalidad por insuficiencia renal crónica 2012-2013. Subdirección de Evidencia de Promoción a la Salud, Base de datos electrónicos.

Thévenod R.B. 2010. Novel aspects of cadmium transport in mammalian cells. In Biometals. 23: 857-875. https://doi.org/10.1007/s10534-010-9309Treviño B.A. 2004. Insuficiencia renal crónica: enfermedad emergente, catastrófica y por ello prioritaria. Cir Ciruj, 72: 3-4 http://www.medigraphic.com/pdfs/circir/c c-2004/cc041a.pdf

USRDS. 2009. Annual Data Report. United States: Atlas of Chronic Kidney Disease \& End-Stage Renal Disease. 
Copyright (c) 2018 Guillerm ina G arcia Juárez, Maricela Hernández Vázquez, Herm ila Orozco B ol años,

Gerardo Suárez González, Efrén Ramirez Bribiesca y Maria Guadalupe Juárez Ortiz

\title{
(c) $(1)$
}

Este tex to está protegido por una licencia licencia CreativeCommons 4.0.

\begin{abstract}
Usted es libre para Compartir —copiar y redistribuir el material en cualquier medio o form ato—y Adaptar el documento —remezclar, transformar y crear a partir del material- para cualquier propósito, incluso para fines comerciales, siempre que cumpla la condición de:

Atribución: Usted debe dar crédito a la obra original de manera adecuada, proporcionar un enlace a la licencia, e in dicar si se han realizado cam bios. Puede hacerlo en cualquier form a razonable, pero no de form a tal que sugiera que tiene el apoyo del licenciante o 10 recibe por el uso que hace de la obra.

Resumenclelicencia - Textocompletocielalicencia
\end{abstract}

